\title{
Ueber die Natur der Nerventhätigkeit.
}

\author{
Von
}

AIrr. Lehmann (in Kopenhagen).

(Mit 4 Textfiguren.)

Es wird jetzt wohl allgemein angenommen, dass der "Nerven-

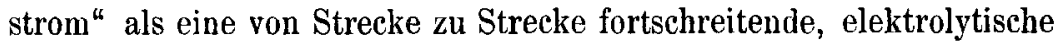
Umlagerung der Ionen aufgefasst werden muss. Die Bedingungen des Zustandekommens einer solchen Bewegung habe ich in einer früheren Arbeit folgendermaassen formulirt. "In einem durchaus gleichförmigen Elektrolyt wird eine solche Bewegung nicht zu Stande kommen können; zwischen zwei sich berührenden Stellen ist ein Unterschied der Concentration erforderlich. Wird dieser hergestellt, so entsteht hierdurch ein elektrischer Potentialunterschied, der nach Zustandekommen des Stromes Energie aus der Stelle mit höherer, nach der Stelle mit niedrigerer Concentration führen wird, bis der Unterschied der Concentration aufgehoben ist. Von der Grösse der auf diese Weise entstandenen elektromotorischen Kraft wissen wir, dass sie dem $\log \left(\frac{C}{c}\right)$ proportional ist, indem $C$ und $c$ die Concentration der beiden Stellen oder die hierdurch bestimmten osmotischen Drucke bezeichnen. Von diesen Thatsachen aus scheint der Nervenprocess sich ohne Schwierigkeit erklären zu lassen. Die Reizung eines peripheren Nervenendes spaltet die chemischen Verbindungen im Nerv; es entsteht hierdurch ein Concentrationsunterschied zwischeh der erregten Stelle und der unmittelbar anstossenden Strecke, der einen Potentialuntersehied herbeiführt. In einem leitenden Organ wie einem Nerv wird dieser Unterschied schwerlich ohne Ausladung der Elektricität bestehen können, und es entsteht mithin ein elektrolytischer Strom. Die elektrische Ausladung geschieht wahrscheinlich eben zwischen den beiden Punkten, die einen Potentialunterschied bekommen haben. Die unter dem Namen Elektrotonus bekannte Erscheinung, die sich an einem unorganischen Elektrolyte 
mit verschiedener Leitungsfähigkeit des Kerns und der Peripherie völlig nachahmen lässt, zeigt uns nämlich, dass in einer Strecke eines Nervs gleichzeitig Ströme in entgegengesetzter Richtung verlaufen können. Folglich verwehrt uns Nichts, in einem Nerv einen geschlossenen Stromkreis zwischen zwei an einander grenzenden Stellen anzunehmen, die einen Potentialunterschied haben. Wegen des elektrolytischen Stroms wird aber nothwendiger Weise die Concentration an der zweiten Stelle vermindert, so dass nun zwischen dieser und der dritten, näher am Centrum gelegenen Stelle ein Potentialunterschied entsteht $u$. s. w. Auf diese Weise muss die Bewegung sich aus der erregten Stelle durch die ganze Nervenleitung hindurch bis in's Centralorgan fortpflanzen." 1)

Diese Theorie hat vor andern, weniger geuau formulirten jedenfalls den Vorzug, dass ihre Gültigkeit sich experimentell prüfen lässt. Da die Theorie nämlich ausschliesslich mit bekannten physikalischen

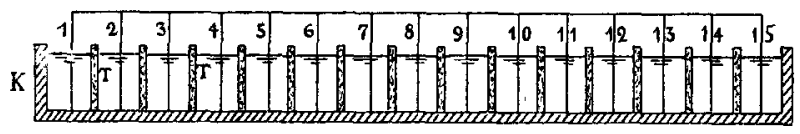

Fig. 1.

Verhältnissen rechnet, wird man leicht untersuchen können, ob ein Apparat, der den Bau eines Nerven nachahmt, auch die bekannten elektrischen Erscheinungen der Nerven hervorbringen kann. Wie wir im Folgenden sehen werden, scheint dies in der That völlig zuzutreffen. Ich werde nun zuvörderst den Apparat beschreiben, und danach einige daran angestellten Messungen mittheilen. Durch diese sind die elektrischen Verhältnisse des Apparates vollständig hestimmt, so dass die bekannten Gesetze der Nerventhätigkeit einfach daraus abgeleitet werden können.

Der Apparat, in Fig. 1 sehematisch dargestellt, besteht aus einem hölzernen Kasten $K, 50 \mathrm{~cm}$ lang, $5 \mathrm{~cm}$ breit und tief, inwendig mit einem starken Schellacküberzug versehen. Der Kasten ist durch poröse Thonwände, $T T$, in 15 Abtheilungen getheilt; die Thonplatten sind in Rinnen im Boden und in den Wänden des Kastens eingefügt und schliessen hier wasserdicht. Man kann einen vollständig dichten Verschluss erreichen, wenn die Rinnen mit

1) Die physischen Aequivalente der Bewusstseinserscheinungen S. 181. 
sehr dickem Schellackfirniss gefüllt und die Thonplatten in den noch feuchten Schellack eingedrückt werden. Besonders die beiden äussersten Abtheilungen, 1 und 15, müssen von den Nachbarabtheilungen so getrennt sein, dass sie leer gehalten werden können, während die ubrigen Abtheilungen gefüllt sind. Dies kann am sichersten dadurch erreicht werden, dass man die beiden äussersten Thonplatten entfernt und statt derselben an jedem Ende des Kastens einen kleinen Thoneylinder, wie sie in den galvanischen Elementen gebraucht werden, einsetzt; diese bilden dann die Abtheilungen 1 und 15. Der Apparat wird mit einer $65 \%$ haltigen Lösung von Zinksulfat gefüllt. Diese Flüssigkeit ist, der oben dargestellten Theorie zu Folge, der Haupttheil des Apparates, weil die elektromotorischen Kräfte durch ihre Concentrationsunterschiede bedingt sind. Die Flüssigkeit muss daher als der "Achsencylinder" des künstlichen Nerven bezeichnet werden, weil einerseits der Achsencylinder der durchaus unentbehrliche Theil eines Nerven ist, während er andererseits auch vollständig genügt, um die Functionen eines Nerven auszuführen. Der Kasten ist die Primitivscheirle unseres künstlichen Nerven; die 'Thonplatten stellen die Ranvier'schen Schnürringe dar, haben aber im Apparate nur den Zweck, Strömungen der Flüssigkeit zu verhindern, und sind desshalb eigentlich unnöthig, wenn man den Kasten mit Schwämmen oder dergleichen indifferenten, porösen Stoffen füllt. Für unsere folgenden Untersuchungen ist indessen die Theilung des Raumes in bestimmt begrenzte Abtheilungen zweckmässiger. Wir werden aber später sehen, dass eben so gut wie der complicirte Apparat ein künstlicher, nackter und hüllenloser Achsencylinder als Nerv functioniren kann.

Im Apparate fehlt jetzt nur noch die Markscheide. Diese könnte zwar aus einem flüssigen Leiter hergestellt werden, der leichteren Handhabung wegen habe ich aber Metall (Zink) genommen, und weil auch die Form unwesentlich ist, nehme ich keinen die Flüssigkeit umgebenden Mantel, sondern ziehe es vor, dem Leiter die in Fig. 1 gezeigte Form zu geben. In jede Abtheilung taucht man eine $3 \mathrm{~cm}$ breite Zinkplatte, oben mit einer Klemmschraube versehen; die Platten sind mit einander in Verbindung gesetzt durch kleine Rollen von Kupferdraht, deren jede $50 \mathrm{~cm}$ eines $0,5 \mathrm{~mm}$ dicken Drahtes enthält. Bei dieser Anordnung kann die Verbindung leicht an jeder Stelle unterbrochen werden, während ein Galvanometer, behufs Messung der Potentialdifferenz, eingeschaltet wird. 
Hiermit ist der ganze Apparat fertig, und wenn die Zinkplatten frisch geputzt sind und die Zinksulfatlösung überall dieselbe Concentration hat, wird der Apparat - wie ein ungereizter und unversehrter Nerv - völlig stromlos sein. Der oben dargestellten Theorie zu Folge wird nun die Reizung eines Nerven eine Concentrationsverminderung des Stoffes herbeiführen; wir müssen also unseren künstlichen Nerv einfach dadurch reizen können, dass wir irgendwo, 7. B. in Abtheilung 1, eine Zinksulfatlösung geringerer Concentration hineinbringen. Es entsteht dadurch ein elektrischer Strom, der nach und nach Veränderungen im ganzen Nerv zur Folge hat, indem das Potential, von der gereizten Stelle aus, überall sinkt. Ehe wir aber zur näheren Untersuchung dieser Veränderungen übergehen, wird es zweckmässig sein, die Methode zu besprechen, nach welcher man aus der Ablenkung des Galvanometers die Grösse der Potentialdifferenzen berechnen kann.

Als Galvanometer diente ein gewöhnlicher Multiplicator mit astatischem Nadelpaar und 1500 Windungen eines $0,25 \mathrm{~mm}$ dicken Kupferdrahtes; die Dämpfung war eine mittlere. Um diesen Multiplicator als Voltmeter gebrauchen zu können, verfuhr ich folgendermaassen. Fine kleine Concentrationskette, von möglichst genau derselben Grösse wie diejenige der einzelnen Abtheilungen des künstlichen "Nerven", wurde hergestellt. Die Elektroden waren Zinkplatten, $3 \mathrm{~cm}$ breit, die eine war constant mit einer $65 \%$ haltigen Zinksulfatlösung umgeben, um die andere wurỉen nach und nach Lösungen geringerer Concentrationen gebracht. Der Strom geht in der Flüssigkeit von der weniger nach der mehr concentrirten Lösung, und für jedes Concentrationsverhältniss wurde der Strom durch das Galvanometer geschlossen und die Ablenkung abrelesen. Die elektromotorische Kraft $e$ einer solchen Kette ist durch die Formel:

$$
e=1,98 \cdot 10^{-4} \cdot T \cdot \frac{2 V}{n(\bar{U}+\bar{V})} \log \frac{C}{c} \text { Volt }
$$

gegeben, wo $U$ und $V$ die absoluten Beweglichkeiten der Ionen, $n$ die Werthigkeit derselben, $T$ die absolute Temperatur und $C$ und $c$ die Concentrationen der Flüssigkeit bedeuten. Für Zinksulfatlösung bei $18^{\circ} \mathrm{C}$. erhält man ungefähr:

$$
e=0,04 \cdot \log \frac{C}{c} \text { Volt }=40 \cdot \log \frac{C}{c} \text { Millivolt. }
$$

Wenn man also die Concentrationen der Lösungen kennt und die entsprechende Ablenkung der Galvanometernadel beobachtet, lässt 
sich eine Tabelle zusammenstellen, aus welcher die Grösse der elektromotorischen Kraft, die einer gegebenen Excursion des Galvanometers entspricht, ersehen werden kann. Die Resultate einer Reihe solcher Messungen sind in Tab. I angegeben. Für die eine Lösung war $C$ constant $=65 \%$; die Concentration der anderen ist unter $c$ angeführt; $v$ ist die beobachtete Ablenkung des Galvanometers. Ferner sind aufgeführt das Verhältniss $\frac{C}{c}, \log \left(\frac{C}{c}\right)$ und die daraus nach der obigen Gleichung berechnete Grösse der elektromotorischen Kraft in Millivolt ausgedrückt. Werden die elektromotorischen Kräfte z. B. als Abscissen, die entsprechenden Ablenkungen des Galvanometers als Ordinaten abgesetzt, so entsteht eine vollständig glatte Curve, mittelst welcher die Interpolation der zwischenliegenden, nicht gemessenen Grössen mit hinreichender Genauigkeit ausgeführt werden kann.

Tabelle I.

\begin{tabular}{c|c|c|c|c}
\hline \hline$c$ & $v^{0}$ & $\frac{C}{c}$ & $\log \frac{C}{c}$ & $e$ \\
\hline 64,35 & 1 & 1,01 & 0,0043 & 0,17 \\
63,06 & 3,5 & 1,03 & 0,0128 & 0,51 \\
61,80 & 9 & 1,05 & 0,0212 & 0,85 \\
58,71 & 17 & 1,11 & 0,0453 & 1,81 \\
55,77 & 21 & 1,17 & 0,0682 & 2,73 \\
52,98 & 26 & 1,23 & 0,0899 & 3,60 \\
47,68 & 34 & 1,36 & 0,1335 & 5,34 \\
$\mathbf{4 2}, 91$ & 41,5 & 1,51 & 0,1790 & 7,16 \\
38,62 & 46 & 1,68 & 0,2253 & 9,01 \\
32,83 & 56 & 1,98 & 0,2967 & 11,87 \\
27,91 & 61 & 2,33 & 0,3674 & 14,70
\end{tabular}

Die elektromotorischen Kräfte einer solchen Concentrationskette sind, wie ersichtlich, ausserordentlich klein; es muss desshalb Sorge getragen werden, dass die Resultate nicht wegen zufälliger Störungen falsch ausfallen. Die wesentlichsten störenden Ursachen können jedenfalls leicht angegeben werden, und da wir dieselben bei der Untersuchung des künstlichen Nerven wieder antreffen, wird es hier am Platze sein, sie kurz zu erwähnen. Erstens sind die Oberflächen der Elektroden von Bedeutung; die Zinkplatten müssen ganz gleichartig und frisch geputzt sein, weil die gemessent: Ablenkung des Galvanometers sonst nicht nach der obigen Formel in Potentialdifferenzen umgerechnet werden kann. Schon die Veränderungen, welche der Strom durch Ausscheidung und Auflösur:g 
von Metall an den Elektroden hervorbringt, genügen, um eine merkliche elektromotorische Kraft zu Stande zu bringen, wenn die beiden Elektroden in dieselbe Lösung getaucht werden. Zweitens zeigt es sich, dass eine mit Zinksulfatlösung benetzte Zinkplatte, selbst wenn sie nur momentan aus der Lösung gehoben wird, so viel Sauerstoff an ihrer Oberfläche condensirt, dass ein zwar kurz dauernder, aber sehr starker Strom entsteht, welcher immer von der anderen Elektrode nach der sauerstoffhaltigen geht. Sauerstoff-Zinksulfatlösung bringrt also eine viel grössere elektromotorische Kraft hervor als zwei Lösungen von sogar sehr verschiedenen Concentrationen. Es muss also sorgfältig darauf geachtet werden, dass die Zinkplatten ruhig stehen, so dass sie nicht bald in grösserer, bald in geringerer Höhe von der Flüssigkeit umspült werden, in welchem Falle die Messungen durchaus unzuverlässig sind.

Nachdem wir jetzt die Vorsichtsmaassregeln kennen, die genau befolgt werden müssen, können wir zur Untersuchung des künstlichen Nerven übergehen. Der Apparat befindet sich in stromlosem Zustand; Abtheilung 1 und 15 sind vorläufig leer, die übrigen mit $65 \%$ haltiger Zinksulfatlösung gefültt, und die darin gesenkten Zinkplatten mit einander verbunden. Wir reizen jetzt den Nerv, indem wir an einem Ende, z. B. in Abtheilung 1, eine 1\% haltige Zinksulfatlösung eingiessen und die darin stehende Zinkplatte mit Platte 2 in Verbindung bringen. Was darauf im Apparate geschehen wird, lässt sich ohne Schwierigkeit vorhersagen. Es entsteht ein elektrischer Stron, dessen Richtung in der Flüssigkeit aus Abtheilung 1 nach den übrigen Altheilungen geht. Ijeser Strom wird in Abtheilung 1 Auflösung von Zink, in allen übrigen Abtheilungen dagegen Ausscheidung des Metalls herbeiführen; die Concentration der Lösung steigt somit in 1, fällt aber sonst überall. Ia die Stromstärke aber dem Widerstand umgekehrt proportional ist, wird der Strom in Abtheilung 2 stärker als in Abtheilung 3, 4 u. s. w. Da ferner die Menge des ausgeschiedenen Metalls der Stromstärke rlirect proportional ist, muss in Abtheilung 2 viel mehr Metall auf die Elektrode niedergeschlagen werden als in Abtheilung 3 und in den übrigen Abtheilungen. Folglich sinkt die Concentration der Lösung schneller in 3 als in 4, hier wiederum schneller als in 5 u. s. w. Es entstehen somit nach und nach Concentrationsunterschiede zwischen den verschiedenen Abtheilungen, und hierdurch entstehen neue elektromotorische Kräfte. Je zwei an einander grenzende 
Abtheilungen des Apparates bilden also eine Concentrationskette, so dass überall locale Stromkreise zu Stande kommen. Auf diese Weise schreitet die Bewegung von Abtheilung 1 nach 15 vorwärts und wird schliesslich auch in der letzten Abtheilung ein Potentialgefäll herbeiführen.

Dass diese Betrachtung richtig ist, können wir theils durch Beobachtung der Stromrichtung zwisehen zwei an einander grenzenden Abtheilungen, theils durch Messung der elektromotorischen Kräfte des Apparates nachweisen. Fangen wir mit dem ersten Punkte an. Es sei z. B. das Galvanometer zwischen Abtheilung 6 und 7 des stromlosen Apparates eingeschaltet. Wird dann die Reizungslösung in Abtheilung 1 hineingegossen, zeigt das Galvanometer sofort einen Strom, der von Abtheilung 6 durch das Galvanometer nach 7 geht. Dies ist leicht verständlich. Die Platten 6 und 7 bilden ja närnlich Theile der positiven Polplatte; der Strom durch die Flüssigkeit nach Platte 6 ist aber stärker als der Strom nach 7 , weil der letztere einen grösseren Widerstand überwinden muss, und folglich muss ein unipolarer Strom von Abtheilung is durch das Galvanometer nach 7 gehen. Die Stärke dieses Stromes nimmt aber allmählich bis Null ab, was darauf deutet, dass die elektromotorischen Kräfte zwischen Abtheilung 1 und 2 abnehmen, und danach zeigt sich ein stets wachsender Strom entgegengesetzter Richtung; folglich muss jetzt das Potential der Abtheilung 6 kleiner als das der Abtheilung 7 geworden sein. Schalten wir in diesem Moment das Galvanometer zwischen Abtheilung 7 und 8 ein, so zeigt sich bier noch ein schwacher Strom von der ursprünglichen Richtung, also von 7 nach 8 ; in einer gewissen Zeit treten aber hier auch die soeben erwähnten Veränderungen ein, so dass ein stets wachsender Strom entgegengesetzter Richtung entsteht. In einem gegebenen Momente nach der Reizung lässt sich also gewöhnlich im Apparate ein Punkt finden, wo zwei an einander grenzende Abtheilungen entgegengesetzte Stromrichtungen zeigen. In allen dem Reizungsorte näher liegenden Abtheilungen geht der Strom dann durch das Galvanometer nach dem Reizungsort; in den ferner liegenden Abtheilungen ist die Richtung des Stromes umgekehrt. Diese Umkehrung der ursprünglichen Stromrichtung zwischen zwei Abtheilungen ist also das Zeichen, dass sich die Erregung lis in die betreffende Abtheilung fortgepflanzt hat.

Die Beobachtung der Stromrichtung zeigt also, dass die Erregung 
sich vom Reizungsorte langsam fortpflanzt, so dass überall neue elektromotorische Kräfte entstehen. Durch Messung dieser Kräfte können wir ferner leicht nachweisen, dass sie um so grösser sind, je näher die betreffenden Abtheilungen dem Reizungsorte liegen. Zwar können wir nicht gleichzeitig die Potentialunterschiede an zwei verschiedenen Stellen bestimmen, da aber die Menge der Flüssigkeit recht bedeutend ist, dauert es eine geraume Zeit, bis der Zustand einer gegebenen Abtheilung sich merklich verändert; dadurch wird es möglich, ein Bild von dem gesammten Zustand des Apparates in einem gegebenen Momente zu entwerfen, trotzdem die Messungen der Potentialunterschiede eine gewisse Zeit in Anspruch nehmen. Ich habe solche Messungen mehrmals unternommen und immer dieselben Resultate erhalten; die äusserst kleinen Abweichungen liessen sich immer auf die Schwankungen der Zimmertemperatur zurïckführen, wodurch die Grösse der elektromotorischen Kräfte ein wenig beeinflusst wird. In Tab. II gebe ich die Resultate dreier vollständiger Messungen; Reihe I wurde 2 Stunden, Reihe II 4 Stunden und Reihe III 23 Stunden nach der Reizung des "Nerven" ausgeführt. Die Zahlen der obersten Reihe sind die Nummern der Abtheilungen; für jede Abtheilung sind darunter zwei Zahlen angeführt, die obere gibt die Potentialdifferenz zwischen der betreffenden Abtheilung und der rechts stehenden an, die untere Zahl bedeutet das Potentialgefäll der Abtheilung. Wie diese letzteren Zahlen gefunden sind, wird sogleich besprochen werden; wir betrachten zuvörderst die Potentialdifferenzen etwas näher.

Tabelle II.

\begin{tabular}{|c|c|c|c|c|c|c|c|c|c|c|c|c|c|c|}
\hline & 1 & 2 & 3 & 4 & 5 & 6 & 7 & 8 & 9 & 10 & 11 & 12 & 1:3 & 14 \\
\hline 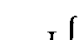 & & 10,9 & 6,5 & 3,0 & 2 & 0,8 & 0,6 & 0,4 & 0,3 & - & - & $\ldots$ & - & \\
\hline 13 & $(72,5)$ & 23,7 & 12,8 & $6, ?$ & $\therefore 3$ & 2.1 & $1,: 3$ & 0,7 & 01.3 & -. & - & - & - & - \\
\hline $\mathrm{II}$ & $(24,0)$ & 11,5 & 8,2 & 5,9 & $\therefore .4$ & 2,11 & 1,4 & 1,2 & 0.9 & 0,7 & 0,5 & 0.3 & 0,2 & - \\
\hline & $(60,2)$ & $: 36,2$ & 24,7 & 16,5 & 10.6 & 7.2 & 5.2 & 3,8 & 2,6 & 1,7 & 1,0 & 0,5 & 0,2 & - \\
\hline IU & 8.5 & 7,1 & 6,5 & 5,4 & 4,5 & 4.0 & 8,3 & 2,8 & 2,4 & 2,0 & 1,6 & 1,2 & 0,4 & 1,0 \\
\hline & 50,7 & 42,2 & 35,1 & 28,6 & 23,2 & 18,7 & 14,7 & 11,4 & 8,6 & 6,2 & 4,2 & 2,6 & 1,4 & 1,0 \\
\hline III & 14,4 & 12,2 & 10,6 & 8.4 & 7,2 & 5,8 & 4,6 & 3,8 & 2,7 & 1.9 & $1, ?$ & 0.7 & 0,6 & 2,2 \\
\hline & 76,4 & 620 & 49,8 & 39.2 & 30.8 & 23,6 & 17,8 & 13,2 & 9,4 & 6.7 & 4.8 & 3,5 & 2,8 & 2,2 \\
\hline
\end{tabular}

In Reihe I, zwei Stunden nach der Reizung ausgeführt, findet man für Abtheilung of die Zahl 0,:3, welche bedeutet, dass zwischen 
Abtheilung 9 und 10 eine elelitromotorische Kraft von der Grösse 0,3 Millivolt gefunden wurde. Für Abtheilung 10-11, 11-12 u. s. w. zeigte das Galvanometer negative Ablenkungen, was den früheren Beobachtungen zu Folge darauf deutet, lass die Erregung sich noch nicht soweit fortgepflanzt batte. Dagegen sieht man, dass die Potentialdifferenz um so grösser wird, je näher dem Reizungsorte die betreffenden Abtheilungen liegen; zwischen Abtheilung 1 und 2 war sie noch so gross, dass sie mit dem zur Verfügung stehenden Galvanometer gar nicht gemessen werden konnte. Aus den Potentialdifferenzen der verschierlenen an einander grenzenden Abtheilungen erhält man das Potentialgefäll einer Abtheilung dadurch, dass man die Differenzen der höheren Abtheilungen summirt. Wenu sich zwischen 9 und 10 eine Differenz 0,3 , zwischen 8 und 9 eine Differenz 0,4 findet, so unuss folglich zwischen 8 und 10 die Differenz $0,3+0,4=0,7$ gefunden werden. Die Zahl 0,7 gibt also an, welche elektromotorische Kraft die Flüssigkeit in Abtheilung 8 mit einer Lösung von der ursprünglichen Concentration geben würde, wenn die beiden Lösungen miteinander in Berübrung gebracht würden, oder mit anderen Worten, die Zahl giebt das Gefäll des elektrischen Potentials der betrefferden Abtheilung an. Auf diese Weise, durch Summation der Potentialdifferenzen sämmtlicher höhpren Abtheilungen, sind die Zahlen der zweiten Reihe bestimmt. Nur für Abtheilung 1, deren Potentialdifferenz nicht gemessen werden konnte, ist die Zahl 72,52 theoretisch abgeleitet. Unter der Voraussetzung, dass die 1\% haltige Lösung in Abtheilung 1 sich in der kurzen Zeit nicht bedeutend verändert hat, muss die elektromotorische Kraft zwischen dieser Lösung und der $65 \%$ haltigen der Abtheilung 10 durch die Formel:

$$
e=40 \log \frac{65}{1}=72,52 \text { Millivolt }
$$

gegeben sein. In Betreff der Reihe II (Tab. 2) ist nur zu bemerken, dass die Zahl 24 für Abtheilung 1 nicht genau gemessen, sondern nur geschätzt werden konnte; sie ist desshalb in Klammer gesetzt, und die ihr anhaftende Uusicherheit geht selbstverständlich in die Zahl der zweiten Reihe $(60,2)$ hinüber.

Die Zahlen der Reihe III sind 23 Stunden nach Anfang der Reizung gefunden; es war nun eine beträchtliche Veränderung in Abtheilung 14 eingetreten. Damit die Grösse der Veränderung bestimmt werden könute, wurde die bisher leere Abtheilung $15 \mathrm{mit}$ 
$65 \%$ haltiger Lösung gefüllt und das Galvanometer zwischen diese und Abtheilung 14 eingeschaltet. Die so gemessene Potentialdifferenz ist für Abtheilung 14 angegeben; die übrigen Differenzen sind wie früher bestimmt, und die Potentialgefälle daraus berechnet. - Die Reihe III a endlich ist nicht die Fortsetzung dieser Messungen, sie wurde bei einer anderen Gelegenheit bestimmt und nur mitgenommen, um die Wirkung einer stärkeren und länger dauernden Reizung zu zeigen. Dies wurde folgendermaassen erreicht. Vier Stunden nach Anfang der

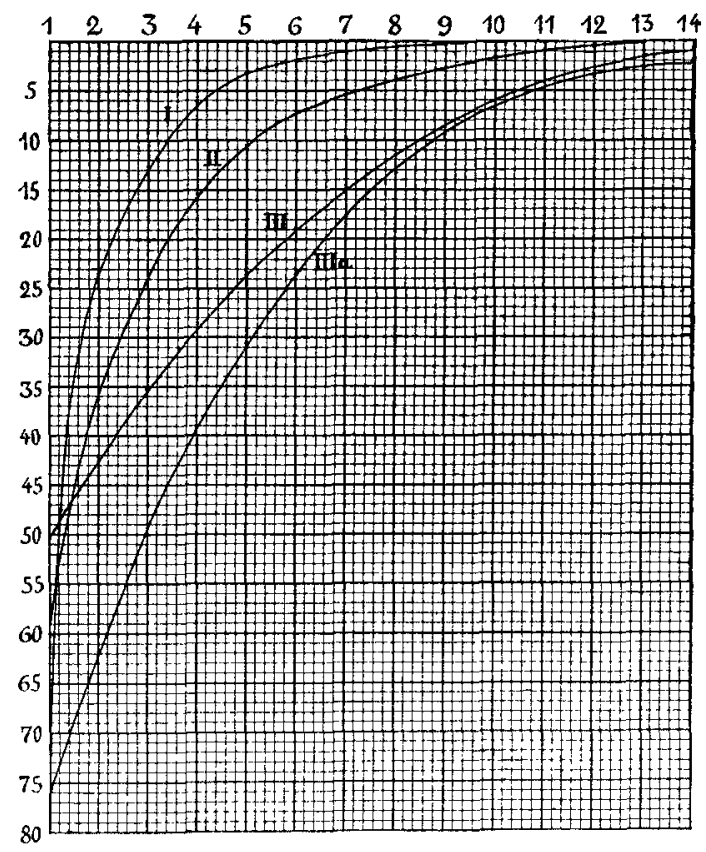

Fig. 2.

Reizung zeigte der Apparat fast denselben Zustand, wie in Reihe II (Tab. II) angegeben. Die Reizungsflüssigkeit wurde nun aus Abtheilung 1 entfernt und eine frische, kaum $1 \%$ haltige Lösung hineingegossen. 20 Stunden nachher wurde der Zustand gemessen; das Resultat ist in Reihe III a angegeben. Die Veränderungen sind, wie ersichtlich, jetzt viel grösser, als sie ohne diese ernenerte Reizung gewesen sein würden; sonst hätten wir nämlich anıähernd die Reihe III gefunden.

Um einen besseren Ueberblick über die Veränderungen im Apparate zu geben, habe ich die Zahlen der Tab. II graphisch dar- 
gestellt in Fig 2. Die Abtheilungen des Apparates sind als Abscissen, die Potentialgefälle, der Natur der Sache gemäss, als negative Ordinaten abgesetzt. Die Curven I bis III zeigen deutlich, wie die Wirkung der Reizung vorwärts schreitet, indem die Concentration der Lösung in den verschiedenen Abtheilungen nach und nach vermindert wirl. während sie in Abtheilung 1 steigt. Ebenso tritt der Unterschierl zwischen III und III a bestimmt hervor.

Nachdem wir somit die Wirkung einer Reizung des künstlichen Nerven kennen, wird es nicht schwer sein, nachzuweisen, dass unser Apparat genau die bekannten elektrisehen Erscheinungen eines wirklichen Nerven zeigt. Erstens sieht man, dass die gereizte Stelle im Verhältniss zu den relativ unveränderten Strecken negativ elektrisch ist; die Bestimmung der Potentialdifferenz an irgend einer Stelle ist ja weiter nichts als eine Messung des ruhenden Nervenstroms, und der Strom geht in solchem Falle stets von der relativ unveränderten Stelle durch das Galvanometer nach dem Ort der Reizung. Ferner zeigen unsere Messungen, dass die Stärke des durch das Galvanometer abgeleiteten Stromes um so grösser wird, je näher dem Reizungsorte und je weiter von einander entfernt die abgeleiteten Stellen liegen. Dies ist auch vollständig in Uebereinstimmung damit, was am wirklichen Nerv beobachtet wird, und ist ein Beweis dafür, dass der Nerv nicht wie ein Metalldraht leitet. Wäre nämlich der Reizungsort allein der Sitz der elektromotorischen Kraft, so müsste die Ableukung der Galvanometernadel durch den Nervenstrom stets proportional der Verlängerung der abgeleiteten Nervenstrecke abnehmen, weil der Widerstand mit der Länge dieser Strecke zunimmt. Die Beobachtungen am wirklichen Nerv zeigen aber, wie bekannt, das Entgegengesetzte, und diese Thatsache lässt sich leicht aus dem Zustand unseres künstlichen Nerven ableiten. Aus Tab. II geht hervor, dass, je weiter man sich von einem willkürlich gewählten Punkte entfernt, das Potentialgefäll um so grösser wird. Folglich muss auch der durch das Galvanometer fliessende Strom um so stärker werden, je länger rlie abgeleitete Nervenstrecke wirl. Und ferner sieht man, dass das Potential in der Nähe des Reizungsortes stärker abfällt als in grösserer Entfernung von diesem Punkte. Daraus folgt einfach, dass einer abgeleiteten Nervenstrecke bestimmter Grösse nicht immer derselbe Galvanometerstrom entspricht: je näher am Reizungsorte, um so stärker wird der Strom. 
Dass diese Folgerungen mit der Erfahrung in Uebereinstimmung sind, können wir leicht durch Messungen bestätigen. Da die Concentrationsunterschiede der verschiedenen Abtheilungen die Ursache der elektromotorischen Kräfte sind, können wir, ohne den Bau des "Nerven" zu stören, einfach durch Ableitung des Stromes die Potentialdifferenzen nachweisen. Wir tauchen z. B. in die beiden $A b$ theilungen 3 und 4 eine frisch geputzte Zinkplatte und klemmen sie am Rande des Kastens fest, so dass sie mit der metallenen Leitung des Apparates nicht in Berührung kommen kann, und wir verbinden diese Flektroden mit dem Galvanometer. Wir erhalten dann einen Strom, der von 3 durch das Galvanometer nach 4 geht, und also derjenigen Stromrichtung entgegengesetzt ist, die wir erhalten hätten, wenn das Galvanometer zwischen den Platten 3 und 4 des Apparates eingeschaltet wäre. Darin ist aber nichts Sonderbares, denn die in Abtheilung 3 eingesenkte Elektrode ist zwar ein negativer Pol der Kette $3-4$, sie ist aber zugleich ein positiver Pol der Kette 2-3, und weil diese letztere die stärkere ist, muss der Strom von Abtheilung 3 durch das Galvanometer nach 4 gehen. Der Multiplicator gibt also unter diesen Umständen den vom Reizungsorte aufsteigenden Strom, den Actionsstrom, an. Wird dagegen das Galvanometer zwischen die Platten 3 und 4 des Apparates eingeschaltet, während sämmtliche übrige Ketten, 1-2, $2-3,4-5$ u. s. f. geschlossen sind, so zeigt die Ablenkung desselben nur den von 4 nach 3 gehenden Strom, den $r$ uhenden Nervenstrom, an. Es ist leicht einzusehen, dass nur dieser letztere Strom an einem wirklichen Nerv direct nachgewiesen werden kann, weil man nicht ohne Verletzung des Nerven an zwei verschiedenen Stellen mit dem Achsencylinder in Verbindung kommen kann; der von dem Reizungsorte aufsteigende Strom, der Actionsstrom, lässt sich daher nur als negative Schwankung des ruhenden Nervenstromes nachweisen. Auf diese Punkte kommen wir später zurück; hier gehen wir nur davon ans, dass die Potentialdifferenzen des Apparates ebensowohl nachgewiesen werden können, wenn wir Elektroden in die Flüssigkeit tauchen und das Galvanometer damit in Verbindung setzen, als wenn wir das Galvanometer in die metallene Leitung einschalten. Wir können also die jedes Mal frisch geputzten Elektroden nach und nach in verschiedene Abtheilungen des Apparates hineinsetzen und für jede neue Stellung die Ablenkung der Galvanometernadel ablesen. Auf diese Weise sind die in Tab. III verzeichneten Resultate erhalten. 
Tabelle III.

\begin{tabular}{|c|c|c|c|c|c|c|c|c|}
\hline & 2 & 3 & 4 & 5 & 6 & 7 & 8 & 9 \\
\hline 3 & 30 & - & - & - & - & - & - & - \\
\hline 4 & 42 & 27 & - & - & - & - & - & - \\
\hline 5 & - & 36 & 23 & - & - & - & - & - \\
\hline 6 & 47 & - & 26 & - & - & - & - & - \\
\hline 7 & - & 44 & 38 & 19 & - & - & - & - \\
\hline 8 & - & - & - & - & - & - & - & - \\
\hline 9 & 48 & 45 & 42 & 29 & - & 15 & - & - \\
\hline 10 & - & - & - & - & - & - & - & - \\
\hline 11 & - & $\overline{-}$ & - & - & - & - & - & - \\
\hline 12 & - & 47 & - & - & - & - & - & 5 \\
\hline
\end{tabular}

Die Zahlen der obersten Reihe und der ersten Colonne links sind die Nummern der Abtheilungen; wo die Colonnen und Reihen sich schneiden, steht die Gradgrösse der Galvanometerablenkung, welche erhalten wurde, wenn die Elektroden in die betreffenden $\mathrm{Ab}$ theilungen getaucht wurden. Da der innere Widerstand des Apparates der gegenseitigen Entfernung der Abtheilungen proportional wächst, können die gemessenen Ablenkungen der Galvanometernadel nicht einfach wie früher in Potentialdifferenzen umgerechnet werden; die Gradgrössen sind also nur ein relatives Maass der Stromstärke. Die Zahlen zeigen aber, dass die Stromstärke, trotz des grösseren inneren Widerstandes, mit der gegenseitigen Entfernung der Elektroden wächst; folglich muss das Potentialgefäll in einem stärkeren Verhältniss wachsen, wie wir es ja auch oben gefunden haben.

Wir können jetzt ohne Schwierigkeit die eigenthümlichen elektrischen Erscheinungen erklären, die ein vom Körper vollständig getrenntes Stück eines Nerven zeigt. Die beiden Schnitte nüssen in diesem Falle als dauernde Reize wirken, und folglich fällt das Potential von der Mitte, dem Aequator, des Nervenstücks nach den beiden Querschnitten ab. Mit dem Apparate lässt sich dieser Zustand leicht nachahmen, wenn wir ihn, von dem ursprünglichen stromlosen Zustand ausgehend, gleichzeitig an beiden Enden gleich stark reizen. Die eintretenden Veränderungen werden durch Fig. 2 veranschaulicht, wenn man die äusserste Linie rechts als Symmetrieachse nimmt und sich eine mit der Zeichnung symmetrische Figur hinzugefügt denkt. Die Abtheilung 14 wird dann der Aequator des Nervenstücks, und von hier an fällt das Potential nach den beideu 
Querschnitten, wie die Curven zeigen. Der von einem solchen Nervenstück abgeleitete Strom wird also um so stärker, je näher eine Elektrode dem Aequator und die andere dem Querschnitte liegen; es ist dabei gleichgültig, ob die Elektroden sich auf derselben Seite oder auf verschiedenen Seiten des Aequators befinden. Werden dagegen Punkte berührt, die symmetrisch um den Aequator liegen, so zeigen sie sich stromlos. Dies alles gilt, wie bekannt, von einem wirklichen Nerv und lässt sich sehr leicht mit dem künstlichen nachahmen.

Es ist ferner leicht verständlich, dass wir mittelst des Apparates den Actionsstrom als eine negative Stromschwankung nachweisen können. Gehen wir davon aus, dass unser künstlicher Nerv sich in dem Zustande befindet, den ein wirklicher Nerv sofort annimmt, wenn er blossgelegt und irgendwo durchschnitten wurde; die elektrischen Verhältnisse dieses Zustandes werden am besten durch Curve III (Fig. 2) veranschaulicht. Wird das Galvanometer jetzt z. B. zwischen Abtheilung 9 und 8 eingeschaltet, so gibt die Ablenkung desselben den ruhenden Nervenstrom an. Reizen wir darauf den Nerv an einem mehr central liegenden Punkt, indem wir in Abtheilung 15 eine verdünnte Zinksulfatlösung hringen, dann treten von diesem Punkte aus dıeselbeu Verändertingen im Nerv ein, wie früher durch die Reizung in Abtheilung $1^{1}$ ). Nach einer gewissen Zeit sinkt also das Potential in Abtheilung 9, und der durch das Galvanometer von 9 nach 8 gehende Strom wird folglich geschwächt. Die Ablenkung der Nadel wird mithin kleiner, d. h. es zeigt sich eine negative Stromsthwankung. Diese wird immer grösser, wenn die Reizung in 15 fortgesetzt wird, und kann schliesslich eine vollständige Umkehrung der Stromrichtung herbeiführen. Wird die Reizung dagegen bald unterbrochen, so gleicht sich die negative Schwankung nach und nach aus. Eine viel genauere Bestimmung des Actionsstromes lässt sich indessen durch direkte Messung ausführen, was unten näher hesprochen wird.

Mit dem Elektrotonus brauchen wir uns hier nicht zu beschäftigen. Diese Erscheinung ist schon früher von Hermann,

1) Stellt man den Versuch an, so ist es am besten, zuvörderst die Reizung zu unternehmen und damn das Galvanometer einzuschalten. Die Reizung verursacht namlich sofort störungen des elektrischen Zustandes der metallenen Leitung, die mit der vier später eintretenden negativen Stromschwankung nichts zu thun baben. 
Gruenhagen u. A., mittelst physikalischer Apparate verschiedener Constructionen, die unserem künstlichen Nerv sehr ähnlich sind, nachgeahmt worden. Selbstverständlich wird sie dann auch an unserem Apparate auf verschiedene Weise nachgewiesen werden können; es kommt nur darauf an, dass Stromschleifen von dem reizenden elektrischen Strom in den Galvanometerkreis einbrechen können.

Wir kommen jetzt zu einem besonders wichtigen Punkt: die Fortpflanzungsgeschwindigkeit der Erregung im Nerv. In Betreff derselben ist jedenfalls eine Gesetzmässigkeit constatirt, nämlich, dass die Geschwindigkeit mit der Grösse des angewandten Reizes wächst. Diese Thatsache ist eine einfache Consequenz der im Nerv stattfindenden Veränderungen. Je grösser nämlich der Reiz, um so kleiner wird die Concentration am Reizungsort, und folglich wird die hier erzeugte elektromotorische Kraft um so grösser. Die Zersetzung des decomponiblen Stoffes im Nerv ist aber, ceteris paribus, dieser ursprünglichen elektromotorischen Kraft direct proportional. In einer gegebenen Entfernung vom Reizungsort sinkt das Potential also um so schneller, je grösser die durch die Reizung erzeugte elektromotorische Kraft ist, oder mit anderen Worten: die Fortpflanzungsgeschwindigkeit der Erregung wächst init der Grösse des Reizes. Von der Richtigkeit dieser Betrachtung kann man sich leicht durch einen Versuch mit dem künstlichen Nerv überzeugen. Nimmt man statt der $1 \%$ haltigen Reizungslösung eine $16 \%$ haltige, wodurch die elektromotorische Kraft gerade 3 Mal kleiner wird, so dauert es mindestens $3 \mathrm{Mal}$ länger, bis sich eine Potentialdifferenz in einem gegebenen Punkte nachweisen lässt. Durch Messung der Fortpflanzungsgeschwindigkeit bei verschiedener Concentration der Reizungslösung kann man ohne Schwierigkeit die Abhängigkeit der Geschwindigkeit von der elektromotorischen Kraft ermitteln. Ich habe jedoch solche Messungen nicht angestellt, weil die Messungen Valentin's am Nerv nicht die für einen Vergleich nöthigen Daten enthalten. Valentin gibt nämlich zwar die gefundene Geschwindigkeit in Metern an, die Reizstärke aber nur durch den Rollenabstand des Inductionsapparates, und hieraus lässt sich ein bestimmtes Maass für die Reizstärke kaum ableiten. Ausserdem schwankt die für eine gegebene Reizstärke gemessene Geschwindigkeit so stark mit dem

1) Moleschott's Untersuchungen zur Naturlehre Bd. 10 S. 520 ff. 1870 . 
jeweiligen Zustande des Nerven, dass von dem Ermitteln eines bestimmten Abhängigkeitsverhältnisses nicht die Rede sein kann. Sicher bleibt nur, dass die Geschwindigkeit mit der Reizstärke wächst.

Aus dieser Thatsache folgt ferner, dass die Erregung sich mit abnehmender Geschwindigkeit fortpflanzen muss. Wenn die Erregung nämlich einen gewissen Punkt erreicht hat, kann die hier erzeugte Potentialdifferenz als Reiz für die folgende Strecke betrachtet werdeu. Da aber diese Potentialdifferenz nothwendig geringer sein muss, als die am Reizungsort erzeugte elektromotorische Kraft, wird auch die Geschwindigkeit der Erregung in der folgenden Strecke geringer als in der ersten, weil -- wie wir gesehen haben - die Fortpflanzungsgeschwindigkeit mit der Reizgrösse abnimmt. Dass es sich bei dem künstlichen Nerv wirklich so verhält, kann leicht nachgewiesen werden; wie sich aber der natürliche Nerv in dieser Beziehung verbält, ist zur Zeit wohl noch unentschieden. Der Nachweis M unk's, dass die Geschwindigkeit um so kleiner ausfällt, je läıger die durchlaufene Strecke ist ${ }^{1}$ ), hat sich durch spätere Untersuchungen nicht bestätigt. R. du B ois-Reymond fand als Mittel einer sehr grossen Anzahl von Messungen, dass die Geschwindigkeit überall dieselbe ist ${ }^{2}$ ). Diese Messungen sind aber nach der elektromagnetiseben Methode angestellt, wo die zufälligen Fehler der einzelnen Beobachtung recht erhehlich sind, jedenfalls viel mal grösser als die kleine Zeitdifferenz, die im günstigsten Falle erwartet werden kann. Nur ausnahmsweise ergehen die Messungen für die verschiedenen Nervenstrecken eine constante Geschwindigkeit; sie nimmt entweder ab oder zu. Die Unsicherheit der einzelnen Messung zeigt sich am besten dadurch, dass zuweilen sogar negative Fortpflanzungszeiten vorkommen. Es scheint mir höchst zweifelhaft, ob man aus einem Beobachtungsmaterial, das mit so grossen zufälligen Fehlern hehaftet ist, wirklich ein zuverlässiges Resultat ableiten kann; die Möglichkeit ist jedenfalls nicht ausgeschlossen, dass eine kleine Veränderung der Geschwindigkeit durch nicht ausgeglichene Fehler verschleiert wird. Nun wird aber das Resultat du Bois-Reymond's durch die nach der graphischen Methode ausgeführten Messungen von Engelmann bestätigt ${ }^{3}$ ). Diese Uebereinstimnung scheint

1) Archiv für Anat. u. Phys. 1860 S. $798 \mathrm{ff}$.

2) Archiv für Anat. u. Phys., Physiol. Abth. 1900 Suppl. s. 97.

3) Archiv für Anat. u. Phys., Physiol. Abth. 1901 S. 1 ff. 
mir aber auch nicht vollständig überzeugend. Engelmann misst nämlich die Geschwindigkeit nicht zwischen den Punkten der Abscisse, wo die Zuckungseurven sich erheben, sondern in einer Höhe vou 1-3 $\mathrm{mm}$ resp. 5-15 $\mathrm{mm}$ über der Abscisse. Die Messungen wurden nicht auf der Abscisse ausgeführt, weil die Zuckungscurven hier einen so spitzen Winkel mit der Abscissenlinie bilden, dass die Anfangspunkte der Curven überhaupt nicht bestimmt werden können. Dieser Unstand ist aber sehr bedenklich, denn die Geschwindigkeit der Nervenerregung kann nur zwischen den Anfangspunkten der Zuckungscurven gemessen werden, während der fernere Verlauf dieser Curven in dieser Beziehung fast gleichgültig ist. Ausserdem zeigen die photographischen Reproductionen der Engelmann'schen Curven, dass ihre gegenseitige Lage in der Nähe der Abscisse sehr schwankend ist, so dass nichts uns Gewähr leistet, dass man aus der gegenseitigen Entfernung der Curven oberhalb der Abscisse auf die relative Lage der Anfangspunkte schliessen kann. Die Frage ist also, meines Erachtens, noch ganz unentschieden, und es würde mich sehr wundern, wenn der natürliche Nerv sich in dieser einzelnen Beziehung von dem künstlichen unterschiede.

Mittelst unseres Apparates kann nämlich sehr leicht nachgewiesen werden, dass die Erregung sich mit abnehmender Geschwindigkeit fortpflanzt. Für diese Messungen ist es durchaus unerlässlich, dass die einzelnen Abtheilungen des Apparates wasserdicht geschlossen sind, so dass die Flüssigkeit nur durch Diffusion durch die Thonplatte aus einer Abtheilung in die andere gelangen kann. Sonst wird nämlich eine Concentrationsverminderung in einer Abtheilung eine Strömung der Flüssigkeit aus der Nachbarabtheilung zur Folge haben, und daraus resultirt, dass die Concentration in beiden Abtheilungen ungefähr gleich schnell sinkt. Wäre also das Galvanometer zwischen diese beiden Abtheilungen eingeschaltet, so würde die Nadel gar nicht abgelenkt, wenn auch das Potential bedeutend gefallen wäre und die Erregung sich schon längst weiter fortgepflanzt hätte. Unter solchen Umständen wäre es also durchaus unmöglich, die Fortpflanzungsgeschwindigkeit in den verschiedenen Strecken zu messen. Ferner muss man sich selbstverständlich vergewissern, dass der Apparat vor der Reizung vollständig stromlos ist. Dies ist nicht immer leicht zu erreichen, wenn der Apparat mehrmals benutzt worden ist, weil gewöhnlich etwas Zinksulfat in die Thonplatten auskrystallisirt und sich wieder auflöst, wenn der Apparat mit frischer 
Zinksulfatlösung gefüllt wird; die Thonplatten müssen daher mindestens 24 Stunden ausgewaschen und nachher getrocknet werden. Wenn diese Vorsichtsmaassregeln berücksichtigt werden, lässt sich die Messung leicht ausführen. Dass die Erregung eine bestimmte Abtlıeilung erreicht hat, erkennt man, wie schon früher (S. 154) dargestellt, daran, dass die Richtung des ursprünglichen Stromes zwischen dieser Abtheilung und der nächst höheren sich umkehrt.

Man braucht hiernach also nur das Galvanometer zwischen zwei Abtheilungen des Apparates, z. B. 2 und 3, einzuschalten und den Moment zu beobachten, wo die Richtung der Ablenkimg der Nadel sich umkehrt. Dann wird das Galvanometer zwischen zwei folgende Abtheilungen eingeschaltet, u. s. f. Auf diese Weise habe ich mehrmals die Fortpflanzunssgeschwindigkeit der Frregung, mit im Ganzen recht übereinstimmenden Resultaten, bestimmt. Nur die Zeit, welche verstreicht, bis das Potential der Abtheilung 2 so weit gefallen ist, dass der Strom von 3 nach 2 geht, ist berleutenderen Schwankungen unterworfen, weil sie von der Beschaffenheit der Reizungslösung abhängig ist. Je mehr Luft (Sauerstoff) diese Flüssiokeit enthält, um so kleiner wird die elektromotorische Kraft zwischen der Reizungslösung und der coucentrirten Lösung des Apparates, und um so langsamer sinkt folglich das Potential. Durch die chenische Thätigkeit der Kette wird aber der Sauerstoff nach und nach verbraucht, und wenn dies geschehen ist, so arbeitet die Reizungslösung, als ob sie von Anfang an luftfrei gewesen wäre. Es ist somit verständlich, dass ein Sauerstoffgehalt der Reizungslösung eigentlich nur eine Verzögerung der Reizung zur Folge hat, und daher von dem Augenblick an, wo die Reizung in voller Stärke eingetreten ist, keine Einwirkung auf die weitere Fortptlanzung der Erregung ausüben kaun. Da aber der Sauerstoffgehalt der Reizungslösung, wenn man nicht besondere Maassregeln trifft, sehr verschieden sein kann, wird auch die Zeit sehr verschieden ausfallen, welche die Fortpflanzung der Erregung bis zu Abtheilung 2 in Anspruch nimmt. Die gefundenen Fortpflanzungszeiten gehen übrigens aus Tab. IV hervor.

Tabelle IV.

\begin{tabular}{l|l|l|l|l|l|l|r|r}
\hline Fortpflanzungszeit & $1-2$ & $2-4$ & $4-6$ & $6-8$ & $8-10$ & $10-12$ & $12-13$ \\
\hline Zwischen den Abth. & bis 40 & 4 & 7 & 12 & 22 & 40 & 93 \\
Vom Anfang der Reizung & - & 44 & 51 & 63 & 85 & 125 & 218
\end{tabular}


Die Zahlen der ersten Reihe geben die Abtheilungen des Apparates an, die darunter stehenden Zahlen sind die Fortpflanzungszeiten zwischen den verschiedenen Abtheilungen in Minuten. Nach dem eben Angeführten kann diese Zeit zwischen Abtheilung 1 und 2 sehr verschieden ausfallen; von Abtheilung 2 ab wächst die Zeit aber recht regelmässig, indem sie für zwei fernere Abtheilungen ungefähr doppelt so gross wie für die soeben zurückgelegten wird. Die unterste Reihe endlich gibt die ganze Zeit an, die vom Anfang der Reizung verstreicht, bis die Erregung die letzte der obenstehenden Abtheilungen erreicht hat.

Die Fortpflanzungsgeschwindigkeit ist also, wie ersichtlich, ausserordentlich gering. Mehrere verschiedene Ursachen tragen hierzu bei. Erstens ist die Stoffmenge, die in jeder einzelnen Abtheilung decomponirt werden muss, um eine messbare Potentialdifferenz hervorzurufen, recht bedeutend. Zweitens ist der Widerstand in der durch viele poröse Thonwände getbeilten Flüssigkeitssäule beträchtlich, so dass die Stromstärke nur gering wird, und folglich wird die pro Zeiteinheit decomponirte Stoffmenge auch nur klein. Endlich sind die oben gemessenen Zeiten eigentlich nicht die wahren Fortpflanzungszeiten. Die Decomposition des Stoffes in irgend einer Abtheilung, z. B. 8, muss schon weit fortgeschritten sein, wenn das Galvanometer einen Strom von 9 nach 8 anzeigt; die Erregung hat thatsächlich lange vorher die Abtheilung 8 erreicht. Wäre es von Interesse, eine grössere Geschwindigkeit zu Stande zu bringen, liesse sich dies leicht dadurch erreichen, dass sowohl die Stoffmenge als der innere Widerstand des Apparates gleichzeitig verkleinert würde, indem man statt Zinkplatten, wie ich sie gebraucht habe, Zinkklötze nimmt, die einen grösseren Theil der einzelnen Abtheilungen ausfüllen. Dies scheint mir indess recht unwesentlich, weil die Bedeutung des Apparates ja gar nicht darin liegt, dass man möglicher Weise durch Kunstgriffe irgend einer Art dieselbe Geschwindigkeit der Erregung wie im wirklichen Nerv zu Stande bringen könnte.

In anderen Beziehungen kann es jedoch von Interesse sein, dass die Processe nicht zu langsam verlaufen, und ich habe es desshalb versucht, die porösen Thonwände zu vermeiden, weil sie unzweifelhaft den inneren Widerstand des Apparates besonders erhöben. Da die Flüssigkeit nicht in Strömungen kommen darf, muss sie von einem indifferenten, porösen Stoff absorbirt werden, und nach verschiedenen Versuchen habe ich gefunden, dass ein baumwollener Docht von der 
Art, die gewöhnlich in Petroleumlampen angewandt wird, sich für unsere Zwecke besonders eignet. Hierdurch lässt sich der Apparat in eine viel einfachere und bequemere Form bringen, wie sie in Fig. 3 dargestellt ist. Ein Dreifuss trägt eine starke Glasplatte $C$; hierauf ruht ein Zinkstreifen $B$, ungefähr $60 \mathrm{~cm}$ lang und $5 \mathrm{~cm}$ breit. Auf dem Zinkstreifen liegt wieder ein baumwollener, $3 \mathrm{~cm}$ breiter Docht $A$, mit Zinksulfatlösung getränkt; dieser Docht stellt also einen hüllenlosen Achsencylinder vor. Das umgebogene Ende des Zinkstreifens mit dem getränkten Docht taucht in ein Glas hinab, und die Reizung des "Nerven" geschieht einfach dadurch, dass Wasser in's Glas gegossen wird. Als Elektroden dieneu gebogene Zinkstreifen $\boldsymbol{E}$,

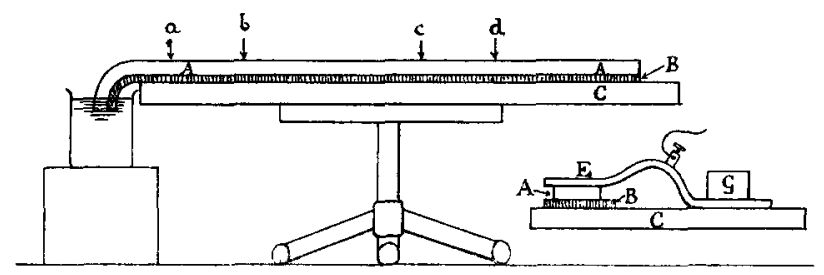

Fig. 3.

ca. $8 \mathrm{~mm}$ breit, die mittelst eines Gewichtes $G$ oder einer Klemme gegen die Glasplatte gedrückt und so festgehalten werden (s. Fig. 3 unten, wo ein Quersehnitt des Apparates gezeichnet ist). Werden zwei solche Elektroden, an den Docht gedrückt, mit dem Galvanometer in Verbindung gesetzt, kann man den ganzen Verlauf des Actionsstromes in den betrefienden Punkten messen.

Dies liess sich mit dem früheren Apparate nicht erreichen, weil es gar zu lange dauerte. Betrachtet man Tab. II, so sieht man, (lass die Potentialdifferenzen, z. B. zwischen Abtheilung 3 und 4, nach und nach die folgenden Werthe annehmen: 6,5 (Reihe I), 8,2 (Reibe II), i,5 (Reihe III). Nun sind die drei Reihen von Messungen resp. 2, 4 und 23 Stunden nach Anfang der Reizung ausgeführt. Ungefähr 3) Stunden hat es also gedauert, bis eine merkliche Abnahme der Potentialdifferenz zwischen diesen beiden Punkten nachgewiesen werden konnte. Durch die fortgesetzte Thätigkeit des Apparates wird diese Differenz, wie wir wissen, bis Null sinken; es würde aher wahrscheinlich andere 24 Stunden in Anspruch nehmen, bis dies erreicht wäre. Durch diesen langsamen Verlauf werden die Messungen praktisch undurchführbar, ganz davon abgesehen, dass sie 
ausserdem unzuverlässig werden, weil Störungen, von Veränderungen der Temperatur, Verdampfung der Flüssigkeit u. s. w. herrührend, unvermeidlich sind. Mit der eben besprochenen neuen Form des Apparates können diese Messungen dagegen in wenigen Stunden ausgeführt werden, und sie sind desswegen von grosser Bedeutung, weil sie mit den Messungen des Actionsstromes am wirklichen Nerv vollständig übereinstimmen.

An zwei verschiedenen Stellen, $a-b$ und $c-d$ (Fig. 3) habe ich solche Messungen ausgeführt. Die Entfernung zwischen $a$ und $b$, sowie zwischen $c$ und $d$, ist $5 \mathrm{~cm} ; a$ ist $5 \mathrm{~cm}, c 17 \mathrm{~cm}$ vom Reizungsorte entfernt. Die Elektroden werden z: B. bei $a$ und $b$ angebracht und mit dem Galvanometer in Verbindung gesetzt; die Anordnung zeigt sich dann fast stromlos. Die Reizungsflüssigkeit (Wasser) wird in's Glas gegossen; es treten kleine Schwankunqen der Galvanometernadel ein, die sich aber immer mehr dem Nullpunkte nähert. Nach Verlauf ungefähr einer Stunde, je nach der Beschaffenheit der Reizungsflüssigkeit, hat die Erregung den Punkt $a$ erreicht; die Ablenkung zeigt einen stets wachsenden Strom von $a$ durch das Galvanometer nach $b$ an. In einer Stunde wird das Maximum erreicht, die Stromstärke sinkt dann jäh, geht durch Null, so dass die Richtung des Stromes sich umkehrt, erreicht wieder ein Maximum, um danach langsam bis Null zu sinken. Ganz denselben Verlauf zeigt der Actionsstrom zwischen $c$ und $d$, nur sind die Veränderungen hier lanysamer und die Amplituden kleiner - wegen der geringeren Potentialdifferenzen, die hier vorkommen. Die Resultate der Messungen sind in Tab. $\mathrm{V}$ angegeben. Die Reihe $t$ gibt die Zeit in Minuten an, von dem Momente gerechnet, wo die Reizung eben den Punkt $a$ erreicht hat.

\begin{tabular}{|c|c|c|c|c|c|c|c|c|c|c|}
\hline \multirow[b]{2}{*}{$\begin{array}{l}t \\
a-b \\
c-d\end{array}$} & \multicolumn{10}{|c|}{ Tabelle V. } \\
\hline & $\begin{array}{r}0 \\
0 \\
0\end{array}$ & $\begin{array}{r}2 \\
-1 \\
\ldots\end{array}$ & $\begin{array}{r}4 \\
-2 \\
\ldots\end{array}$ & $\begin{array}{r}7 \\
-3 \\
\ldots\end{array}$ & $\begin{array}{c}11 \\
-4,5 \\
\ldots\end{array}$ & $\begin{array}{r}13 \\
-5 \\
\ldots\end{array}$ & $\begin{array}{r}15 \\
-6 \\
.\end{array}$ & $\begin{array}{r}18 \\
-7 \\
\ldots\end{array}$ & $\begin{array}{r}20 \\
-8 \\
\ldots\end{array}$ & $\begin{array}{c}23 \\
-y \\
\ldots\end{array}$ \\
\hline $\begin{array}{lr}t & \\
a & b \\
c & -d\end{array}$ & $\begin{array}{r}26 \\
-10 \\
\ldots\end{array}$ & $\begin{array}{r}29 \\
-11 \\
.\end{array}$ & $\begin{array}{r}32 \\
-12 \\
\ldots\end{array}$ & $\begin{array}{r}40 \\
-14 \\
. .\end{array}$ & $\begin{array}{r}56 \\
-16 \\
0\end{array}$ & $\begin{array}{r}60 \\
-16 \\
.\end{array}$ & $\begin{array}{c}72 \\
-13,5 \\
. .\end{array}$ & $\begin{array}{r}79 \\
-10 \\
. .\end{array}$ & $\begin{array}{r}84 \\
--7 \\
-8\end{array}$ & $\begin{array}{r}87 \\
-5 \\
\ldots\end{array}$ \\
\hline $\begin{array}{l}t \\
a-b \\
c-d\end{array}$ & $\begin{array}{r}91 \\
-2 \\
\ldots\end{array}$ & $\begin{array}{r}96 \\
0 \\
\ldots\end{array}$ & $\begin{array}{r}99 \\
+\quad 2 \\
\ldots \quad\end{array}$ & $\begin{array}{l}101 \\
+3 \\
\ldots\end{array}$ & $\begin{array}{l}105 \\
+5 \\
\ldots\end{array}$ & $\begin{array}{l}125 \\
-7 \\
-7\end{array}$ & $\begin{array}{r}127 \\
+10 \\
. .\end{array}$ & $\begin{array}{r}145 \\
+12 \\
\ldots\end{array}$ & $\begin{array}{r}163 \\
+14 \\
\ldots\end{array}$ & $\begin{array}{r}190 \\
+11 \\
-9\end{array}$ \\
\hline $\begin{array}{l}t \\
a-b \\
c-d\end{array}$ & $\begin{array}{r}205 \\
+7\end{array}$ & $\begin{array}{c}210 \\
-4\end{array}$ & $\begin{array}{l}215 \\
+4 \\
+2\end{array}$ & $\begin{array}{r}223 \\
+3 \\
\ldots\end{array}$ & $\begin{array}{r}230 \\
+2 \\
. .\end{array}$ & $\begin{array}{r}235 \\
+1 \\
+10\end{array}$ & $\begin{array}{c}240 \\
+0,5 \\
\ldots\end{array}$ & $\begin{array}{r}245 \\
0 \\
\ldots\end{array}$ & . & \\
\hline
\end{tabular}


Die Reiben $a-b$ und $c-d$ geben die Ablenkung der Galvanometernadel in Graden an; bis $18^{\circ}$ kann die Stromstärke als der Ablenkung proportional betrachtet werden. Einen besseren Ueberblick, als die Zahlen gewähren können, erhält man durch Fig. 4, wo die Zeit als Abscisse, die Ablenkungen als Ordinaten abgesetzt sind; die volle Linie entspricht der Reihe $a-b$, die gestrichene Linie der Reihe $c-d$. Die Curven stimmen, wie man sieht, vollständig mit denjenigen überein, die man von grösseren Muskeln oder Nerven mittelst des repetirenden Rheotoms erhalten hat ${ }^{1}$ ). Aus dieser Uebereinstimmung der Wirkungen dürfen wir unzweifelhaft auf gleichartige Ursachen schliessen. Was in dem künstlichen Nerv stattfindet, lässt sich aber leicht angeben.

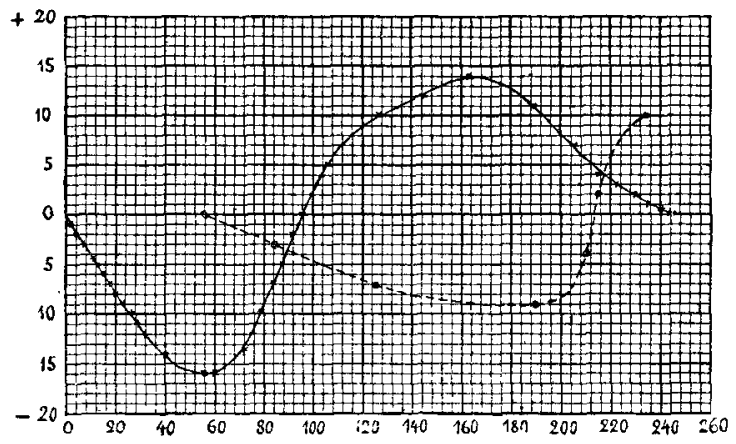

Fig. 4.

Wenn die Erregung sich dem Punkte a nähert, wird die hier liegende Elektrode positiv elektrisch, wegen der vom Reizungsorte ausgehenden Concentrationsverminderung, und folglich geht der Strom von der Elektrode $a$ durch das Galvanometer nach $b$. Beim Fortsehreiten der Erregung wächst die Stromstärke und erreicht ihr Maximum kurz vor dem Momente, wo die Concentration bei $a \mathrm{zu}$ sinken anfängt. Sobald dies geschieht, nuss die Stromstärke jäh sinken. Theils wird nämlich dann die Potentialdifferenz zwischen $a$ und den dem Reizungsorte näher liegenden Punkten geringer, und theils wird $b$ jetzt positiv elektrisch im Verhältniss zu $a$, so dass also ein Strom von $b$ durch das Galvanometer nach $a$ dem ursprünglichen Strome entgegenwirkt. Indem die Erregung sich weiter nach $b$ fortpflanzt, wird der Strom von $b$ stets stärker, so dass sich die beiden Ströme bald aufheben; die Richtung des Stromes kehrt sich

1) Vgl. z. B. Tigerstedt, Physiologie Bd. 2 S. 26. Leipzig 1898. E. Pfluger, Archiv für Physiologie. Bd. 97. 
nun um, und dieser neue Strom erreicht dann sein Maximum, wenn die Concentrationsverminderung in der Nähe von $b$ möglichst gross geworden ist. Danach sinkt die Stromstärke wieder, um Null zu werden, wenn die Concentrationen bei $a$ und $b$ gleich gross geworden sind; dies kann aber erst langsam zu Stande kommen. Die Theorie kann somit den eigenthümlichen Verlauf der beiden Phasen des Actionsstromes vollständig erklären.

Als Resultat dieser verschiedenen Untersuchungen dürfen wir unzweifelhaft behaupten :

Ein lebendiger, in Thätigkeit versetzter Nerv verhält sich in elektrischer Beziehung als eine Reihe an einander grenzender Concentrationsketten, die dadurch zu Stande kommen, dass der Reiz einen Concentrationsunterschied und somit eine elektromotorische Kraft hervorbringt.

Die psychologischen Consequenzen dieser Theorie habe ich schon früher, in meiner oben citirten Arbeit (S. 182-86 und 262-69), eingehend erörtert. 